unable to abstract the schema from patterns with such low Rc.

Conversely, the difference between the $100-100$ and $100-40$ groups on the last 20 patterns could be largely attributed to the limited usefulness of the schema in encoding individual patterns at $40 \% \mathrm{Rc}$.

These results raise an interesting question for further research. Is the inability to abstract schemata from low Rc patterns the result of an ultimate limit imposed by the physiological mechanisms involved in schema learning, or is this a limitation that can be overcome by special training?

An answer could be obtained by giving Ss schematic learning-set training (as in
Edmonds, Evans, \& Mueller, 1966) with high $\mathrm{Rc}$ schema families, then transferring them to a new schema family composed of low Rc patterns. Having "learned how to learn" schemata may enable Ss to abstract schemata even from patterns with $40 \% \mathrm{Rc}$. RFFERENCES

EDMONDS, E. M., FVANS, S. H., \& MLELLER, M. R. Learning how to learn schemata. Psychonomic Science, 1966, 6, 177-178.

FVANS, S. H., \& MUELLER, M. R. VARGUS 9: Computed stimuli for schema research. Psychonomic Science, 1966, 6, 511-512.

MUELLER, M. R., EDMONDS, E. M., \& EVANS, S. H. The quantification of schema theory and its implications for various perceptual tasks. Paper presented at the meeting of the Southwestern Psychological Association, A pril 1966.

\section{The effect of two types of associative linkage and position of association linkage on the recall of sentences}

\author{
JOSEPH W. GALLAGHER and WILLIAM PATTERSON \\ University of Alabama, University, Alabama 35486
}

The present study examined the influence of two types of association data on the recall of sentences. The results showed that "syntactic restricted" associations facilitated the recall of sentences while typical free associative connections may have some influence. However, the predominant underlying variable may be a syntactic association. The position of syntactic associative connection in the sentence had no differential effect on recall.

The present study consists of two experiments. One experiment examined the influence of free-association strength (FAS) collected in the typical manner (Palermo \& Jenkins, 1964) on the recall of sentences. The second experiment was a replication of Experiment 1, except that syntactic associations (SA) were employed as an index of association strength. The syntactic associations were collected by presenting an independent group of Ss with stimuli and asking them to write down a word that follows the stimulus in written or spoken language. The sentences in Experiment 2 had $0 \%$ typical FAS value. In both studies the location of the associative linkage was manipulated. Certain sentences had associative linkage between the adjective-noun (A-N), the noun-verb $(N-V)$, or the verb-adverbial (V-Adb) positions.

Experiment 1 was designed to extend an earlier investigation (Rosenberg, 1966). Rosenberg reported that sentences with a mean FAS $=23.25 \%$ (taken from Palermo \& Jenkins, 1966) were recalled more readily than those with "moderate" associative connections (no value given) and those with a mean FAS $<.01 \%$. Rosenberg (1966) simultaneously employed associative values in each of the three possible positions it could occur, i.e., between the adjective-noun, noun-verb, and verb-adverbial for both the high- and low-value sentences. For the "moderate" value sentences, an association existed only between the last two words (verb-adverbial). Thus, to some extent, associative linkage and position of association in a sentence is confounded.

\section{EXPERIMENT 1 Lists}

Three lists of nine sentences each were constructed. Each sentence consisted of the following: adjective-noun-verbadverbial. In each list three sentences had three highly associated (20\%) word pairs, three sentences had three weakly associated word pairs (4\%), and three sentences had no associated pairs. List 1 , List 2 , and List 3 had the association between the adjective-noun, noun-verb, and verb-adverbial, respectively. The association values between pairs were taken from the Palermo-Jenkins (1964) norms. Subjects

Thirty Ss were employed. All were students in introductory psychology and completed the experiment for extra credit. Apparatus and Procedure

Each $S$ was told that he would be presented with a number of sentences one at a time for a short period of time. He was instructed that after seeing the sentences he would be requested to write down as many words, parts of sentences, or full sentences that he could recall. The $S$ was instructed that this procedure would continue until they correctly recalled all of the sentences.

Each sentence was presented by means of a Lafayette Memory Drum with the anticipation shutter mechanism disconnected so that the $\mathrm{S}$ saw each sentence for $1 \mathrm{sec}$. Following each presentation of the six sentences, the Ss were given a slip of lined paper and instructed to write down any words, parts of sentences, or full sentences they could remember. Maximum recall time was $60 \mathrm{sec}$ per trial; however, since this was usually more than enough time, when the $S$ stopped writing for approximately $5 \mathrm{sec}$, the $E$ asked him if he had written all he could remember. If the S replied "yes," the data sheet was removed and the next trial was given. This procedure was employed so that if the $S$ finished recalling before $60 \mathrm{sec}$ and could see the recall sheet, the $S$ could not rehearse those words and sentences on the recall sheet. All Ss were run until a criterion of two consecutive errorless trials.

Results and Discussion

An analysis of variance was applied to the trials-to-criterion data. The analysis consisted of one between-S factor (location of FAS in the sentence) and one within-S factor (level of FAS). Neither the main effects nor the interaction was significant. This was totally unexpected and would appear to be in disagreement with the results of Rosenberg (1966). However, several major factors should first be noted. An examination of the sentences used by Rosenberg (1966) indicated that all of the high FAS sentences employed were meaningful (i.e., semantically consistent), while for the medium and low FAS sentences, three of four in each group were anomalous. Other investigators have indicated (Miller \& Izard, 1963; Marks \& Miller, 1964) that grammatical, meaningful sentences are dealt with more readily than syntactically correct, but anomalous, sentences. It was anticipated that the differences in the Rosenberg (1966) study may have been, at least in part, due to the lack of semantic consistency, and not FAS. The present study, in employing syntactic 
word pairs taken from typical FAS norms, may not reflect syntactic contiguity, but an addition of meaning for words (Entwistle, 1966). This suggested that typical FAS stimulus-response pairs may not be entirely satisfactory to use as a variable in the study of associative linkage on learning sentences. In view of this, trial-to-criterion analysis was done for each sentence. The results showed that all of the sentences were not learned at the same rate. The sentences could be ordered from easiest to hardest for all three lists. It was hypothesized that some of the word pairs employed may have been associated through contiguous use in the written and spoken language and that syntactic association may not be manifest in a typical FAS test.

All 24 words used in the sentences were then presented to college students with instructions to write the word that "most likely follows the word in written or spoken language." "New syntactic associations" (SA) were calculated for each sentence. It was found that all of the sentences contained syntactic associations that were not found in the Palermo-Jenkins (1964) typical FAS data. The mean trials to three criterion for the sentences with the highest overall syntactic associations (SA) was significantly less than that for three lowest-value sentences for all three lists. The correlation between SA value of the sentences and rate of learning was from $r=.77$ to $r=.84(p<.01)$. These results strongly suggest that in sentence learning one must consider syntactic associations which actually exist but may not be found in a typical free-association type of test.

\section{EXPERIMENT 2}

Since FAS collected in the typical word-association manner and $S A$ as discussed in Experiment 1 were confounded, it was decided to replicate Experiment 1 with only SA employed in certain sentence positions. The purpose of Experiment 2 was to examine the effect of "syntactic associations" collected with new words and on a different population. Sentences were constructed that consisted of no typical FAS values and no SA values, while others were constructed that had only "syntactic association" located in certain positions in the sentences.

\section{Subjects}

Ninety Ss were employed. All were students in introductory psychology and completed the experiment for extra credit. Three groups of $30 \mathrm{Ss}$ each were assigned randomly to one of the experimental groups.

Lists

The lists were essentially the same as in Experiment 1. Each list consisted of six sentences, three associated and three nonassociated. The association values employed were SA associations. These values were taken from data collected prior to the present experiment. One hundred freshmen enrolled in introductory psychology at the University of Alabama were presented with 100 words chosen randomly from the adjectives, nouns, verbs, and adverbs in the Palermo \& Jenkins (1964) norms. Each $S$ was given a booklet with one word printed at the top of each page. Ss were instructed to write a word on the page that they thought most likely would follow the word on the top of the page in written and spoken language. The task was self-paced, but Ss were encouraged to respond as rapidly as possible. Percentage values for words occurring as responses were calculated for each stimulus word. List 1 had SA located only between the A-N, List 2 had SA only between the N.V, and List 3 had SA only between the V-Adb. The mean SA for the associated sentences in Lists 1, 2, and 3 was approximately $18 \%$. All words other than those designated in the experiment had $0 \% \quad \mathrm{SA}$ and $0 \%$ FAS. The nonassociated sentences had $0 \%$ SA and $0 \%$ FAS between all words.

\section{Apparatus and Procedure}

This was identical to that employed in Experiment 1.

\section{Results and Discussion}

An analysis of variance was conducted on the trials-to-criterion data. The analysis consisted of one within-S factor (SA values) and one between-S factor (location of SA in the sentence).

The main effect of $S A$ value was significant $[F(1,58)=42.69, p<.001]$. Individual $\mathrm{t}$ tests showed that for all three lists the associated sentences were learned faster than the nonassociated sentences (List 1, $t=3.48, d f=29, p<.01 ;$ List 2, $t=4.71, d f=29, p<.01 ;$ List $3, t=2.99$, $\mathrm{df}=29, \mathrm{p}<.01)$. The main effect of location of the SA was not significant $[F(2,107)<1, p>.05]$ and the interaction was not significant $[F(2,107)=2.15, p>.05]$

The results of Experiment 2 indicate that SA facilitates the recall of sentences. The SA appears to be a more relevant index of the associative linkage between syntactic word pairs than typical FAS since the sentences employed had 0\% FAS. In examining the normative data collected for use in the present study, it was found that all of the syntactic pairs found in the Palermo-Jenkins (1964) normative data were also in the normative data used in the present study. Thus, it appears that there may be an underlying syntactic associative hierarchy for many words. It appears that this can influence recall. While this hierarchy may be related to that manifest by syntactic pairs found in typical FAS data, the present experiment has indicated that this type of associative linkage can facilitate independently the recall of sentences. In spite of what may be said about the importance of associative connections and language behavior, the fact cannot be ignored that SA does exist and influences performance in at least one of the tasks used to study the possible influences of syntax of behavior.

The present study indicates that in studies investigating the learning of any syntactic unit it is not sufficient to construct nonassociated units using the typical FAS values as an index of association without some control for learned associations.

It is suggested that in studying the associative hierarchies of individuals some attention be given to the fact that several associative hierarchies may exist. Presumably, the dominant hierarchy would be controlled by the immediate situation. Thus, in the use of association values in experiments, what the $\mathrm{E}$ perceives as the functional aspect may be different from that of the S.

The finding that two different association hierarchies (SA and FAS) can exist independently may be of importance when considering the developmental shift of syntactic responding (collected in the typical manner) to paradigmatic responding. It is suggested that since syntactic responding is high in young children (Entwistle, 1966), in the young child the FAS and SA word hierarchies are similar. Thus, the child's contiguous verbal experiences are predominant in formulating his associations. With adults the syntactic associative hierarchy may be secondary and only manifest when the $S$ is forced, via an experimental task, to learn syntactic units.

\section{REFERENCES}

ENTWISTLE, D. R. Word associations of young children. Baltimore: The Johns Hopkins Press, 1966.

MARKS, L. E., \& MILLER, G. A. The role of semantic and syntactic constraints in the memorization of English sentences. Journal of Verbal Learning \& Verbal Behavior, 1964, 3 , 1-5.

MILLER, G. A., \& IZARD, S. Some perceptual consequences of linguistic rules. Journal of Verbal Learning \& Verbal Behavior, 1963, 2, 217-228.

PALERMO, D. S., \& JENKINS, J. J. Word association norms: Grade school through college. Minneapolis: University of Minnesota Press, 1964.

ROSENBERG, S. Recall of sentences as a function of syntactic and associative habit. Journal of Verbal Learning \& Verbal Behavior, 1966, 5, 392-396. 